\title{
Doscientos Setenta Raspados Uterinos para el Aborto
}

\author{
Dr. Gustavo Isaza Mejía
}

Del Departamento de Ginecología y Obstetricia de la Universidad de Antioquia

\section{MATERIAL}

$1^{0}$ - Historias Clínicas: En 100 historias revisadas observamos que la mayoría de las pacientes llegaron al servicio hospitalario cuando ya el aborto era inevitable, es esta la razón por la cual carecen por completo de datos de gran importancia, tales como: historia familiar, social, condiciones económicas, ambiente en que vive, relaciones con su marıdo, reacciones caracteriológicas y afectivas, historia sexual, actitud frente a los hijos, evolución de los embarazos. de los partos, posibles causas de los abortos de acuerdo con la opinión de la paciente y en fin muchos otros datos que constituyen una historia de tipo sicológico; ya que en opinión de la mayoría de los autores consultados, el aborto tiene como causas principales las anomalías del huevo y los trastornos emocionales. Algunos sostienen que las causas emocionales superan el $50 \%$.

En los datos encontrados, merece comentarse el del aborto y su relación con el número de partos.

La mayor frecuencia del aborto se presenta en aquellas madres que ya han tenido tres embarazos $(16 \%)$ cuatro $(10 \%)$ cinco $(12 \%)$. Nos llamó la atención este dato, y lo consideramos de gran importancia como afirmación del párrafo anterior. Naturalmente es la madre de 3,4 ó 5 hijos la que tiene como quien dice el hastío del embarazo, el terror al parto, y a la responsabilidad de una familia numerosa: es la que aborta más fácilmente. 
$2^{\circ}$ - Hallazgos Histológicos en los Abortos: (270 preparaciones).

\section{Cuadro $N^{\circ} 1$}

Restos placentarios con vellosidades: 137: Abortos reales Reacción decidual solamente:

Otros diagnósticos:

71: " dudosos

52: " negativos

Vellosidades normales: (Fig. 1) 64

" anormales: 73

" del primer trimestre: 91

" del segundo trimestre: 30

" del tercer trimestre: 16

Cuadro $N^{o} 2$

ESTROMA :

Con estroma normal: $\quad 64$

Con edema del estroma: (Fig. 2) 33

Con células de Hofbauer: $\quad 63$

Sin células de Hofbauer: $\quad 51$

Capilares normales: $\quad 35$

Vellosidades avasculares: $\quad 47$

Paredes vasculares hiperplásicas: 5

Paredes vasculares normales: 115

TROFOBLASTO:

Langhans normal: $\quad 64$

Langhans atrófico: $\quad 50$

Sincicio con vacuolas: (Fig. 3) 62

Sincicio sin vacuolas: $\quad 52$

Sincicio con granulaciones: (Fig. 4) 18

Sincicio hiperplásico: (Fig. 2) 21 
$3^{\circ}$ - Comentarios.

El dato numérico de las anormalidades encontradas en las vellosidades coriales no coincide exactamente con los totales enunciados. Esto se debe a que la patología de estos elementos es muy polimorfa. El hecho de encontrar alteraciones en unas vellosidades, no significa en forma alguna, que el diagnóstico deba darse como establecido. En la placenta, con más dificultad que en cualquier otro tejido, el diagnóstico no se hace por hallazgos aislados sino que se requiere un estudio en conjunto, como sucede por ejemplo en los infartos. Es necesario hacer un estudio macroscópico muy minucioso de toda la placenta. En otros términos, uno o más infartos de pequeño calibre, en una placenta de apariencia normal, no significa en forma alguna que exista patología que deba tenerse en cuenta para afirmar un diagnóstico.

El $66 \%$ de los abortos corresponde al primer trimestre del embarazo; esto lo interpretamos también como una confirmación de la causa emocional; es en esta época precisamente cuando la madre está en peores condiciones para adaptarse a su nuevo estado y reacciona más fácilmente desencadenando el aborto.

\section{Cuadro $N^{o} 3$}

Espacios Intervellosos:

Con trombosis:

Sin trombosis:

Con hemorragias:

Sin hemorragias:

Infartos:

Trofoblasto Degenerativo:

I Grado:

II "

III "

IV "

Sin infartos:
13 I Grado:

2 II "

1 III "

27 IV

62 Trofoblasto normai:
21

69 
El infarto de la placenta tiene sus grados (Cuadro $\mathrm{N}^{0} 3$ ), desde ligeras formaciones fibrosas (Grado I) hasta verdadera invasión por el tejido conjuntivo y presencia de hemorragias que ahogan por completo las vellosidades (Grado IV).

Cuadro $N^{\circ} 4$

\section{Patología Asociada}

Lues: (Fig. 5)

Eritroblastosis : (Fig. 6)

Mola hidatiforme:

Corioepitelioma :

Embriones: (Figs. 7 y 8)

Inflamación aguda: (Fig. 9)

Inflamación sub-aguda:

Inflamación crónica:

Vellosidades necróticas antiguas:

Huevos sin embrión: (Fig. 10)

Merece comentario el hecho de encontrar tan elevado número de inflamaciones: aguda 44 sub-aguda 24 y crónica 5; gran número de ellas acompañadas de vellosidades normales. Nos hace pensar esto en la posibilidad de aborto criminal lo cual nos fue imposible comprobar debido a la falta de datos clínicos. (Véase Cuadro $N^{0}$ 5).

Ciento veintiocho casos $(47 \%)$ resultaron con diagnóstico diferente al propuesto clínicamente. Presentaron reacción decidual setenta y una preparaciones $(30 \%)$. En ellas no se encontraron las vellosidades, único elemento que autoriza para confirmar el diagnóstico.

Siete casos presentaron muy claramente el endometrio con glándulas en penacho (Signo de Arias Stella), característico del embarazo ectópico que se comprobó posteriormente. 
Otros hallazgos: Total: 128, sin vellosidades

Hemorrágica

Vasos dilatados:
21

23

Reacción decidua! solamente: 69

Con pigmento (hemosiderina): (Fig. 11)

Calcificaciones :

Endometrio en Fase Progestacional:

" Con reacción de Arias Stella: (signo de embarazo ectópico) (Fig. 12)

" En fase estrogénica:

Adenocarcinoma :

Mola hidatiforme:

” Hiperplásico :

Endometritis :

Cuarenta y ocho casos, completamente diferentes. El número tan elevado de fallos en el diagnóstico clínico, en parte se debe, a que la mayoría de ellos fueron hechos de urgencia y por un personal auxiliar poco adiestrado y también porque en realidad la comprobación presenta en la práctica muchas dificultades que nos hacen recomendar cautela para no cometer el error de hacer el diagnóstico de aborto apresuradamente cuando en realidad se trata de otra entidad muy diferente.

\section{CONCLUSIONES}

$1^{0}$ - El diagnóstico de la causa del aborto por la histología, es muy difícil debido al polimorfismo de su arquitectura histológica.

$2^{\text {o }}$ - Debido a la frecuencia del factor sicógeno como produc- 
tor de Abortos, debe hacerse siempre un estudio muy minucioso en este sentido, el cual sería de gran utilidad para un tratamiento profiláctico posterior.

$3^{\circ}$ - En ausencia de signos macroscópicos evidentes de aborto, no debe afirmarse este diagnóstico sin previo estudio histológico. Una ligereza en este sentido puede ocasionar graves consecuencias familiares y sociales.

$4^{9}$ - La presencia de inflamación concomitante con vellosidades normales, permite sospechar el aborto criminal.

$5^{o}$ - El diagnóstico de sexo es posible, si se estudia el porcentaje del cromosoma sexual.

$6^{0}$ - La presencia de glándulas endometriales en forma de penachos (Signo de Arias Stella) autoriza para hacer el diagnóstico de embarazo ectópico.

NOTA: Los casos presentados pertenecen al Servicio de Obstetricia que dirige el Profesor Benicio Gaviria G. y las preparaciones histológicas al Departamento de Patología que dirige el Profesor Alfredo Correa $\mathrm{H}$.

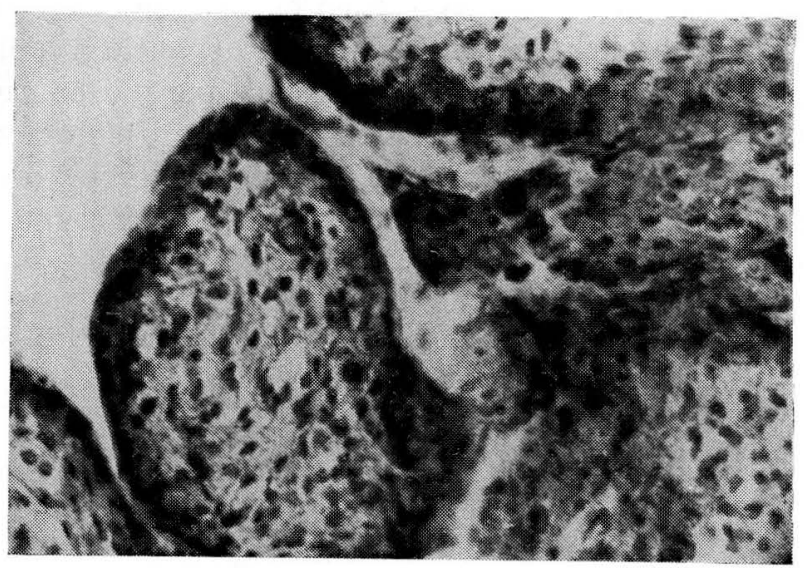

FIGURA No 1 - Vellosidad normal. 


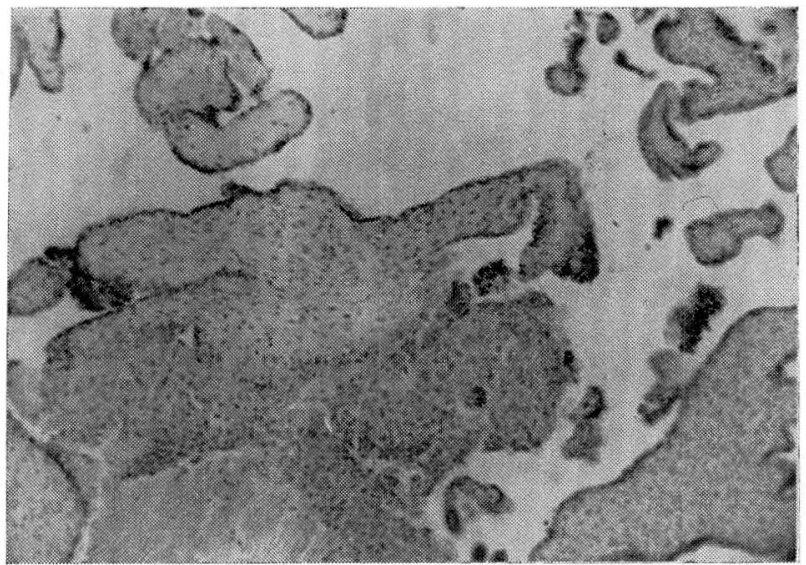

FIGURA No 2 - Edema del estroma. - Hiperplasia corial. Degeneración Micromolar.

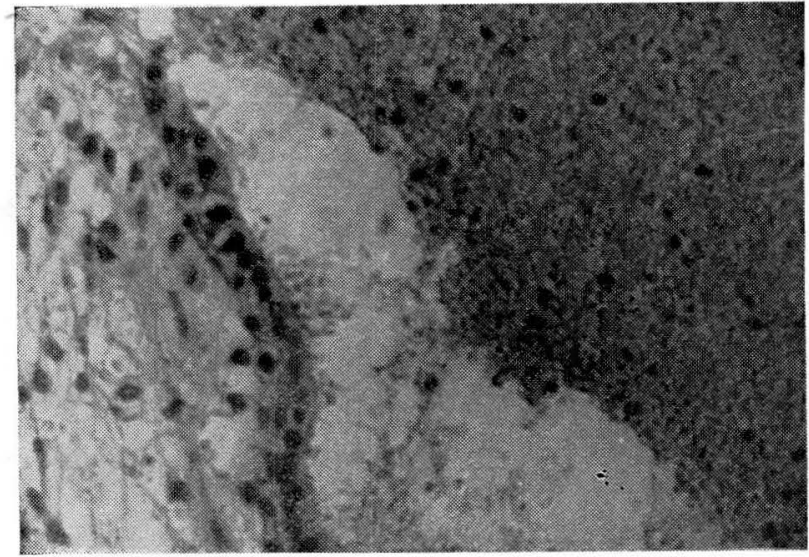

FIGURA No 3 - Vacuolas del Trofoblasto.

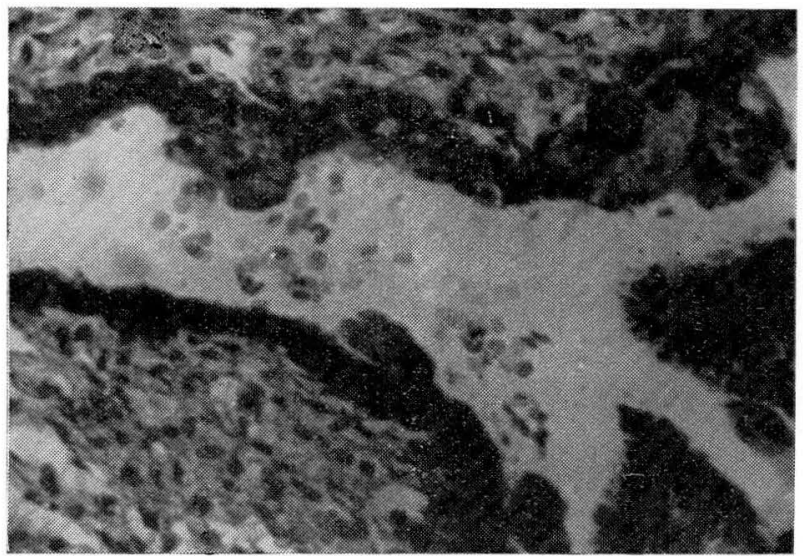

FIGURA No 4 - Granulaciones del Trofoblasto. 


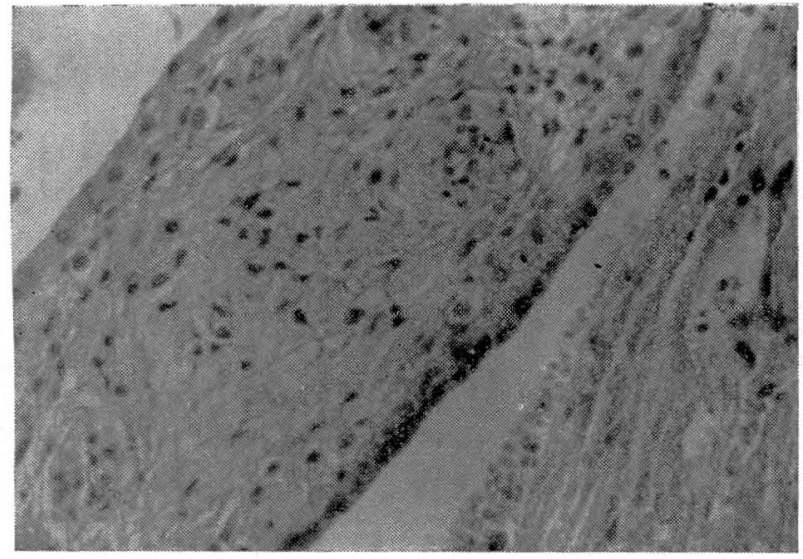

FIGURA Nọ 5 - Vellosidad Fibrosa. - Capilares con paredes infiltradas por Infartos. Sífilis?

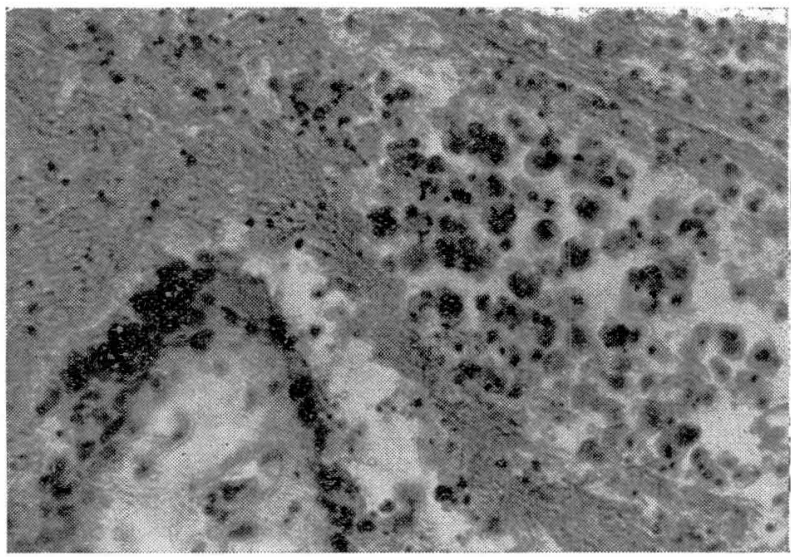

FIGURA № 6 - Eritroblastos intra y extravellositarios. Eritroblastosis.

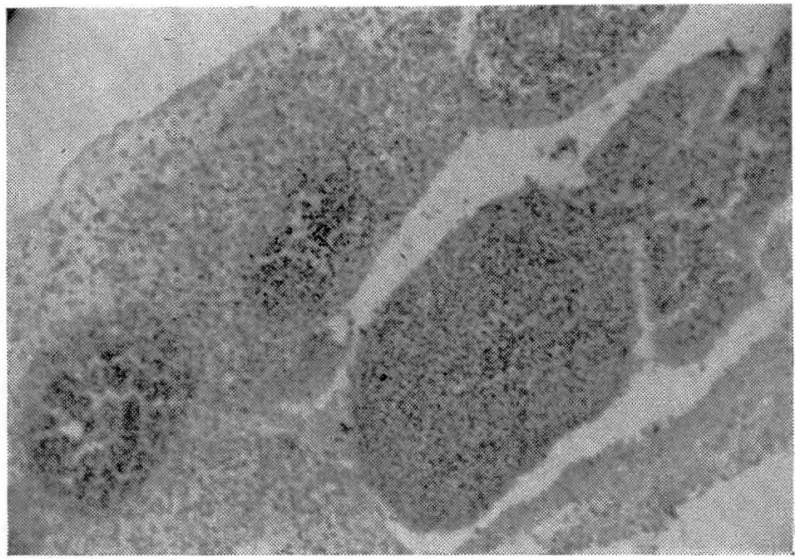

FIGURA Nọ 7 - Embrión de 4 milímetros. 


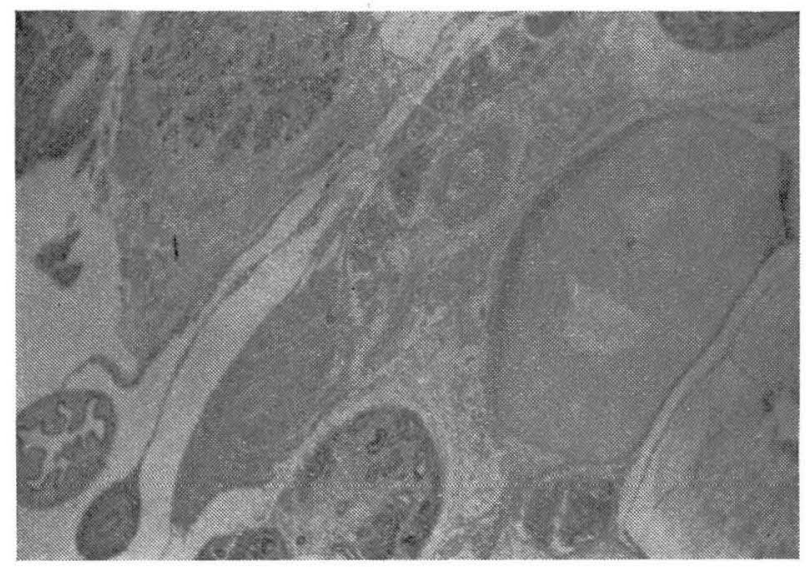

FIGURA N 8 - Embrión de 10 milimetros.

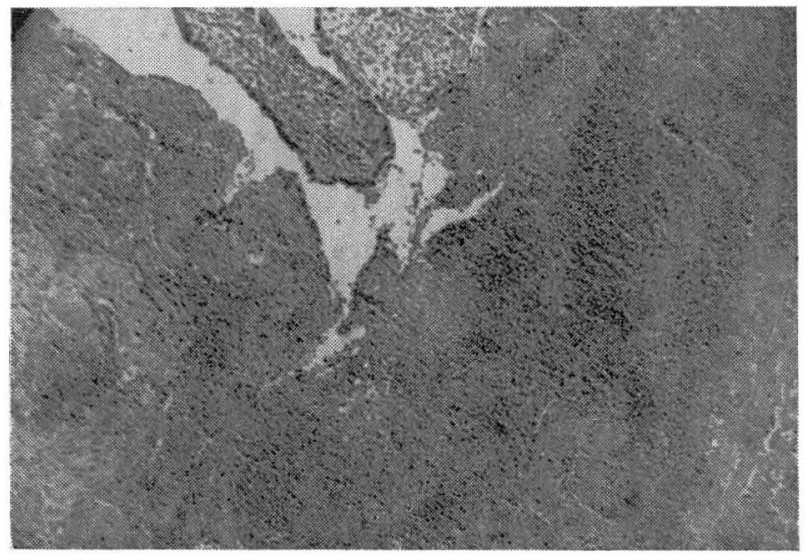

FIGURA № 9 - Inflamación aguda y vellosidades coriales normales. - Aborto criminal.

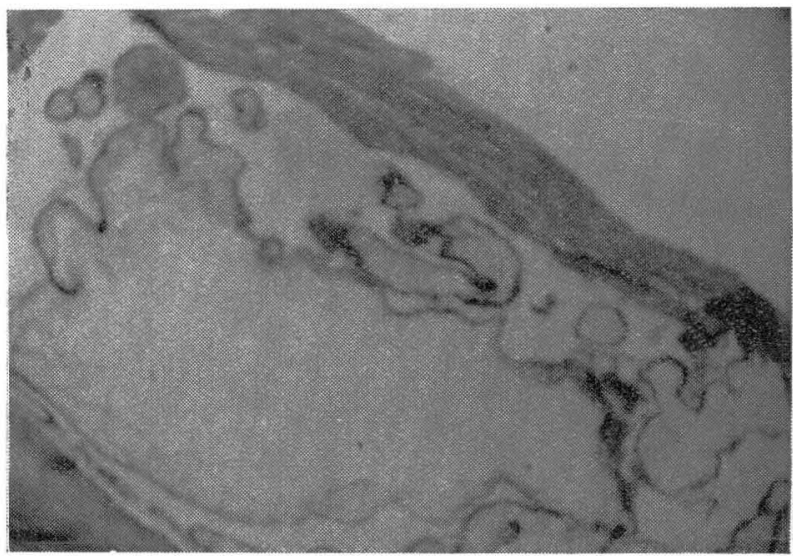

FIGURA № 10 - Huevo sin embrión. 


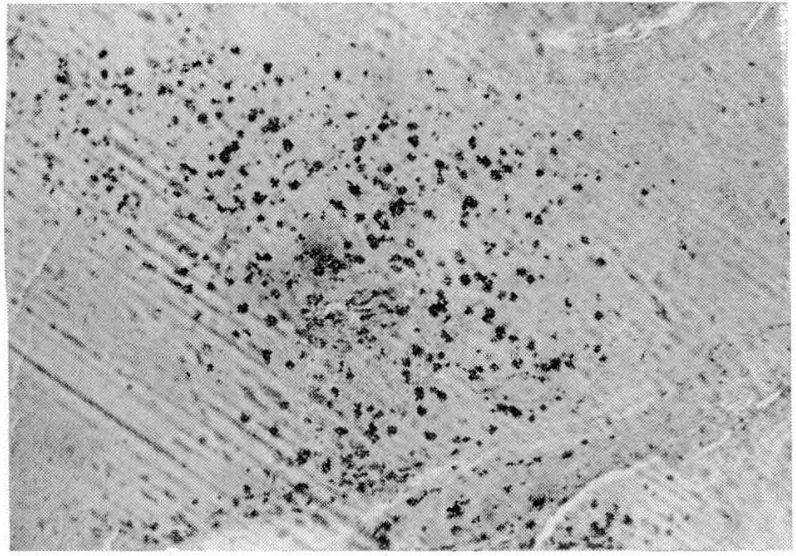

FIGURA Nọ 11 - Pigmento hemático. Hemorragias repetidas y antiguas.

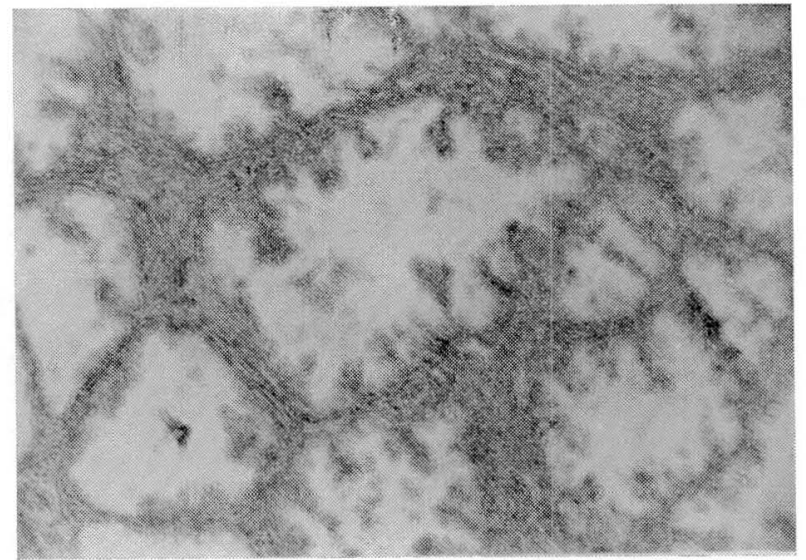

FTGURA No 12 - Glándulas endometriales en penachos "Signo de Arias-Stella". Embarazo Ectópico.

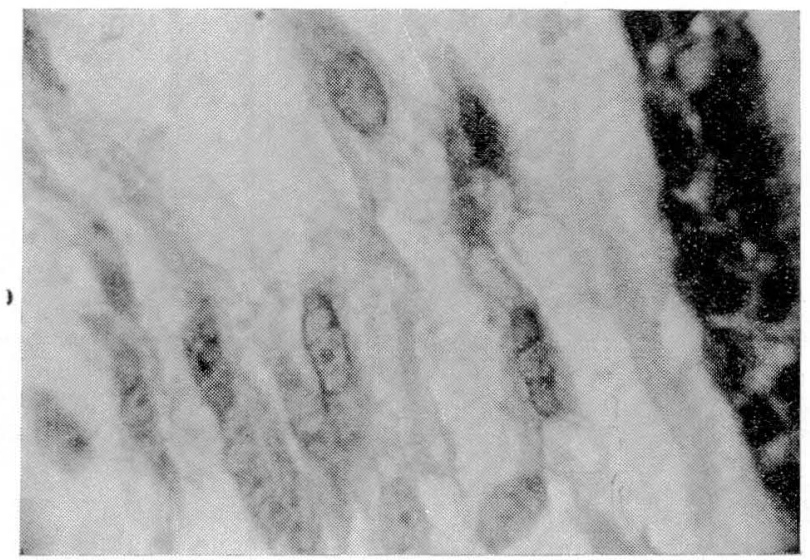

FIGURA No 13 - Cromosoma sexual visible en los núcleos de vellosidad. - S. Femenino. 


\section{BIBLIOGRAFIA}

1) NOVAK: Gynecological and obstetrical Pathology.

2) BOTELLA LLUSIA JOSE: Los ruevos abortivos.

3) G. LEOPARDI. A. ONNIS: Quadri istomorfoligici ed istochimici della placenta Nell idrope fetale. Attualitá di ostetricia e ginecologia. 1956.

4) G. BARTOLOMEI, A. ONNIS: La placenta nella gravidanza Prolungata. Attualitá di ostetricia e ginecologia. 1957.

5) LENNART NILSSON: Hydatidiform Degeneration in Aborted ova. Goteborg. 1957.

6) BOTELLA LLUSIA J. SUAREZ CAMARGO J.: Investigaciones sobre la placenta en el aborto. 